\title{
Physics architecture. Part two
}

\author{
Nelly Konopleva ${ }^{1, *}$ \\ 1"VNIIEM Corporation” JC, Horomnyi tupic 4, 107078 Moscow, Russia
}

\begin{abstract}
Physics architecture. Part two.
The talk is devoted to the jubilee of academician M.A. Markov, who was born in 1908. Now we have also the 100th anniversary of the gauge invariance principle proposed by Weyl in 1918. During the period from 1967 to 1988 M.A. Markov was Academician Secretary of Nuclear Physics Department of AN USSR. He organized the series of conferences dedicated to the topical problems of theoretical, nuclear and elementary particle physics. One of them was international seminar "Vector mesons and electromagnetic interactions" under A.M. Baldin direct leadership. It went off in JINR in September of 1969. This very that seminar gave birth to the conferences series named "Baldin autumn". Thanks to the discussions at the A.M. Baldin seminar many critical situations in theoretical and experimental physics were subsequently settled.

The first plenary session of the seminar included L.D. Faddeev talk about gauge field quantization, which cleared the way to develop the renormalized quantum gauge field theory. My talk was dedicated to the transformation of the classical gauge field theory into geometrical one. As a result the unified geometrical theory was obtained, which could include any interactions together with Einsteinian gravity. In this connection both formulations of Weyl's local gauge invariance principle (of 1918 and 1929) as well as Einsteinian principle of general relativity were used. This approach was based on the fibre bundle geometry, which was developed by mathematicians at that time. This theory gives a realizable classification of interactions according to the local Lie's symmetry groups associated with them. This approach leads to the VIth Hilbert's problem solution.

To obtain further development of the gauge field theory, it is necessary to decide the question of the gauge field mass origin. It is the key moment both in quantum and geometrical classical theories of the gauge fields. Therefore it is very important to analyze the experiments, where massive and massless particles are converted in each other (similar Baldin experiments in JINR in 1967).
\end{abstract}

\section{Introduction}

In the autumn of 1969 in his opening speech of the Baldin International Seminar academician M.A. Markov talked [1]: "... year by year NPD of AN USSR carries out short series of seminars on separate narrow problems of nuclear physics. Last year the seminars on CPT-invariance, neutrino physics, fireballs were organized; within this year the seminar on axiomatic physics has been carried out, today seminar "Vector mesons and electromagnetic interactions" is at work."

\footnotetext{
*e-mail: nelly@theor.jinr.ru
} 
"Why was the vector meson subject chosen this time?

As you know, some years ago the process appeared which can be named as vectorization of elementary particle physics. In the weak interaction theory in accordance with experiment vector and pseudovector variants asserted. The intermediate vector meson idea arose. YangMills fields idea appeared, which got subsequent development in the results of Sacurai and other theorists. A sort of vector domination appeared in the hadron physics.

This specific role of vector interactions is defined by the fact, that different fields of those kinds are related with the conservation laws of the sources of these fields, with the conservation laws of corresponding currents ..."

"All these reasons have made some halo of exclusiveness around vector interactions. Apparently the time has come to tally up these ideas and understand which from them are successful and which are not justified.

It is necessary to note, that vector interactions are measurably distinguished not only in the microworld. In ultramacroworld the very important role remains with them. The point is that vector fields are incompatible with the closed metric (for instance, Friedman metric), if the total charge of vector field sources is nonzero in the world. Vector fields (for instance, $\rho$-, $\omega$ - and $\phi$-fields) can discontinue the collapse of celestial bodies [1]".

\section{Vector fields and unification of fundamental interactions}

The time has confirmed the fundamental role of the vector interactions not only in nuclear physics and electrodynamics, but also in physics of cosmos. In description of vector interactions, really, the key concept is the conservation laws and the vector fields of Yang-Mills kind. The fundamental role of the local gauge invariance was found as principally new point of the theory construction. This is invariance to the symmetry group, whose transformation parameters are the function depending on the space-time point. The simplest example of this kind symmetry is the gauge invariance of the second kind in electrodynamics noted by Maxwell. Similar invariance of the Maxwell equations under analogous group of the gauge transformations with number parameters (gauge invariance of the first kind) is related with the electric charge conservation law. But where is the gauge invariance of second kind leading to it was obscure for a long time.

Yang-Mills equations were formulated in 1954 to describe the elementary particle weak interactions. They are nonlinear generalization of Maxwell equations for electrodynamics. It was expected that after quantization they would give a description of behavior of vector meson triplets carrying weak interactions. But in 1963 Feynman found that in contrast with electrodynamics the quantization procedure proposed by him in 1948 could not be used for Yang-Mills fields quantization because of its nonlinearity [2]. Therefore for a long time it was usual thing to say that Yang-Mills theory would never become a literally physical theory. In 1965 the Nobel Prize in physics was awarded to Feynman. However Feynman was mistaken. Soon many gauge models became be quantizated and renormalized. As a result the experimental discoveries appeared.

In 1967 B. de Witt in USA [3] and L.D. Faddeev and V.N. Popov in USSR [4] realized quantization of Yang-Mills fields by improved Feynman's method - path integral formalism. Just these results were reported by L.D. Faddeev at the Baldin Seminar of 1969 in Dubna. Other proposed approaches turned out of less importance than quantum theory of Yang-Mills fields.

In 1967 for the first time the renormalizable unified theory of weak and electromagnetic interactions was constructed. It was the Weinberg-Salam model [5]. Neutral currents predicted by this model were experimentally discovered in 1973 (Nobel prize of 1979). In 1974 
heavy $j / \psi$-particles were experimentally found. It was a new kind of particles - charmed particles (the Nobel Prize of 1976). One of the authors of this discovery - S. Ting - participated in the Baldin seminar of 1969.

In 80 'es $W$ - and Z-bosons, which are carriers of weak interactions were experimentally found (the Nobel Prize of 1984). In 1990 the Nobel Prize was received by the group of scientists, whose experimental investigations promoted quark models of elementary particles. In 1999 G. t'Hooft and M. Veltman was awarded with the Nobel Prize for massive WeinbergSalam model renormalization [6].

In 1961 M.Gell-Mann was the first to apply the local $S U(3)$-symmetry for classification of the strongly interacting particles $[7,8]$ and to construct the strong interaction theory similar to Yang-Mills one. In 1969 he was awarded with the Nobel Prize for his work. In 1979 S.L. Glashow became the Nobel Prizeman also (for his neutral current prediction).

This incomplete enumeration of Nobel Prizes shows that in 70th -90th of XX century the appearance of the gauge field theory led to the change of the microworld picture. There appeared the Standard Model of elementary particle interactions and Quantum chromodynamics. "Baldin autumn" 1969 opened a new page in nuclear and elementary particle physics.

After the Weinberg-Salam model and Gell-mann and Glashow works [8] the Standard Model was proposed. The Standard Model describes not only electromagnetic and weak interactions, but already includes strong interaction theory - Quantum Chromodynamics. Thus, unique approach was found to three from four fundamental interactions. Gravity remains outside of the Standard Model. But it is not simply to add it there, because gravity is closely related with the space-time structure and properties of the Universe. Moreover gravity is not described by vector-potential. Its field variable is a symmetric tensor of rank two, which is simultaneously the space-time metric. It is only clear that under construction of the gravitation theory, which will combined with the Standard Model, it is necessary to apply the same principles of invariance and symmetry, which were used during the Standard Model construction. In this model different interactions form some hierarchy according to the symmetry groups of the corresponding theories. So, Lie group U(1) corresponds to electrodynamics, Lie group $\mathrm{SU}(2)$ corresponds to weak interactions (Yang-Mills fields), Lie group SU(3) corresponds to chromodynamics. The similar hierarchy of coupling constants arises from the group hierarchy. The coupling constants increase simultaneously with the group dimension. But a prediction exists that under very high energies all coupling constants will become equal. Where will gravity be at that time? The Standard Model does not give any answer.

\section{Gauge fields and geometry. Einstein problem}

Nowadays the local symmetry groups are said to be the gauge group symmetry, and the vector fields, which are transforming by means of their operations are said to be the gauge fields. These titles are not connected with the physical nature of the corresponding interactions. They indicate only that the mathematical structure of the theory of each from them is similar to that of the other. This is realization of the idea of the unified theory of all fundamental interactions on the base of symmetry principles. It is found that this approach makes possible to include, naturally, Einsteinian gravity to the number of the gauge fields $[9,10]$.

In this scheme Einsteinian gravity becomes having the same rights with the vector gauge fields, if the following points are chosen: 1) the local gauge group is the group of arbitrary continuous coordinate transformations of 4D space-time; 2) field variable is a symmetric tensor of rank two. Evidently, these postulates coincide with Einsteinian axioms of General Relativity (GR). The group of arbitrary continuous coordinate transformations of 4D spacetime can be regarded as local symmetry group of Special Relativity (SR). Thus, the relation between GR and SR becomes clear. This way leads to generalization of the vector gauge field 
theory to the tensor gauge fields, because it is not necessary that the symmetric tensor of rank two was identified with space-time metric. The obtained equations will be universal for all tensors of this kind $[9,10]$.

Universality of the approach outlined above permits to consider in the geometric aspect not only gravity, but vector interactions also. To formulate mathematically this point of view, it is necessary to make use of fiber bundle space geometry. This geometry was developed by mathematicians in 50'es - 60'es years of XX century. This very approach was reported by me at the Baldin Seminar of 1969 in Dubna [1]. After this seminar I published the book, including my formulation of the classical Lagrangian and geometric theories of the gauge fields (the Chapters 1 - 3), and quantum theory of these fields formulated by V.N. Popov (the Chapter 4). Thus the contents of the first Baldin autumn plenary session was reflected in our book (V.N.Popov is coauthor of L.D. Faddeev). This book was published in 1972 [11] with M.A. Markov and A.M. Baldin supporting (Atomizdat, Moscow). It turned out the first monograph containing the full gauge field theory and became the manual for many scientists in many countries. The second edition followed first one [12]. It was translated into English in 1981 [13]. The third edition was published in Russian in Moscow in 2000 [14].

The geometric description of all the fundamental interactions, including Einsteinian gravity, opens the way to construct a unified geometric theory of them. Perhaps, we shall know if theory quantization can be replaceable by its geometrization. This is very the question, which worryed Einstein for a long time.

The above-mentioned problem was the topic of the day in well-known discussion between Einstein and Bohr during 30 years. Why Einstein did not recognize quantum mechanics? He did not assume that proper physical theory can be based on probability theory. Einstein was looking for the way of different interactions theories unification across extension of spacetime geometry and properties of geometrical objects. Fiber bundle space geometry gives just the solution of some problems of this kind [13].

\section{What is the structure of the gauge field theory?}

\subsection{0th anniversary of the Weyl's local gauge invariance principle}

After Einsteinian gravitation theory was formulated in 1916 [15], Weyl proposed his theory in 1918. It geometrically unified gravity and electromagnetism [16]. In General Relativity the gravity interaction is related with arbitrary continuous coordinate transformations (general relativity principle). Weyl proposed to add to this theory once more principle - invariance under local gauge transformations of interval $d s^{2}$. In his opinion the new invariance principle must lead to a new field appearance. Weyl identified this field with electromagnetic one, because he found that in his theory the new field was described by means of Maxwell equations. But Weyl's theory was subjected to destructive criticism of Einstein, and Weyl gave up it.

In 1929 Weyl returned to the idea of the local gauge invariance in connection with the problem of electron motion in external fields [17]. He wanted to pass from the free electron motion to the electron motion in the new external field by means of the local invariance principle introduction into the Dirac electron theory. Then it was required the invariance of Dirac's Lagrangian under the local gauge transformations of the electron wave function $\psi^{\prime}$ $=\exp ^{i e \alpha(x)} \psi$. In order to ensure such invariance, Weyl introduced a new field to Dirac's theory. The vector-potential of this field $A_{\mu}$ was transforming to $A_{\mu}^{\prime}=A_{\mu}-\partial_{\mu} \alpha(x)$ (Maxwell gradient invariance of second kind), when the electron wave function was transforming as it was mentioned. The induced by Weyl's postulate of the invariance new equations described the electron motion in the external electromagnetic field. They coincided with the Maxwell 
equations. Theorists did not receive any new information about the electrons and their motions by means of the Weyl's approach. Therefore they began to consider it as curios case. V. Pauli severely criticized Weyl's theory [18].

Serious investigations of the local invariance role in construction of the field theory began only in 60ies of XX century. It was the result of the weak interactions experiments and YangMills fields. It was found that Weyl's method of unification of gravity and electromagnetism can be realized in the Finsler geometry, where in each point of space exists an additional line beginning from this point. Finsler geometry is the simplest case of fibre bundle space geometry.

\subsection{0th anniversary of the Nöther's theorem [19]}

Gauge field theory is constructed from "the first principles" similar to geometry. Symmetry and invariance principles are the basic positions of this theory. In a similar manner geometry of homogeneous spaces was constructed by S.Lie and F. Klein [20]. In 1872 F.Klein proposed the new approach to geometry. He made use of the S. Lie's group theory of space transformations (motions), which Lie developed a short time before. Klein proposed to consider geometry the theory of invariants of Lie group, which transforms the given space into itself. Then all the geometric objects located in this space would be characterized by sets of the given Lie group invariants.

Now the similar situation exists in relativistic and elementary particle physics. The elementary particles are considered to be located and moving objects in the Minkowski space, which is a homogeneous space. Its symmetry group is Lorentz group (one from Lie groups of global symmetry). The objects being investigated are characterized by the sets of invariants of Lorentz group and its representations. Lorentz group (and its extension - Poincaré group) is the base of relativistic invariance principle in the special relativity. Therefore, elementary particle classifications are closely connected with classifications of Lorentz and Poincaré groups representations.

Usually we think of geometrical objects as static figures. Without external action they can conserve their location for a long time. Elementary particles, conversely, are usually represented as moving in some space. In the case of classical theory of this motion its dynamics is described by the equations obtained by means of the variational principle of minimality of the action integral $S$. The solutions of these equations, which are ensuring action minimality, are said to be called extremals. On extremals conservation laws are fulfilled.

The conservation laws, which characterize the particles motion, flow from variational principle also. The varying value in this case is the action integral $S$ again. But now we take no interest in extremum of the action integral. We wish to know the symmetry properties of this integral. E. Nöther investigated the symmetry and invariance properties of the action integral $S$ under space-time coordinate transformations. In 1918 she proved two very important theorems. The first theorem is relative to the case of global symmetry, and the second theorem is relative to the case of local symmetry.

\subsection{Equations of motion, conservation laws and Nöther identities}

In the classical mechanics and field theory the lines, which are the trajectories of motion of the body's mass centre (i.e. only one point of lengthy body!) and light beams, are determined by the motion equations (or field equations). They are the extremals of the action integral $S$. They are also called geodesic lines. 
If the theory (i.e. $S$ ) is globally symmetric, the variable number in these equations is equal to the number of equations. This equation system can be solved under the given boundary conditions.

If the theory (i.e. $S$ ) is locally symmetric, the variable number in the equations is less than the equation number. This equation system can not be solved without covering of additional conditions on the variables. This very situation appears in the gauge field theory and GR. Thanks to overdetermination of the corresponding equation system we can consider some of them following from remaining one. Usually it is convenient to obtain the motion equations of particles as follow from the field equations together with Einstein equations. Then the particles can be regarded as singular points of the field lines.

If the theory is developed in the globally symmetric space-time, the number and form of the conservation laws are determined by the first Nöether theorem. The number of conservation laws coincide with the number of Lie group symmetry invariants. All the known differential conservation laws (energy, momentum, impulse ets.) are given by the first Nöther theorem. The invariant values destined for the experimental testing are obtained by integration of the differential conservation laws arising from the above-mentioned first theorem and its generalization on the Lie's groups of the global internal symmetry. The transformations of such groups depend on the number parameters. This generalization was obtained by me in $1967[10,11]$.

It is necessary to note that the first Nöther theorem and its generalization are only fulfilled in the case of island distribution of the matter.

In the locally symmetric theories the conservation laws obtained by the first Nöther theorem and its generalization are absent. More exactly, they become trivial identities $0=0$ on the extremals.

Disappearance of the energy-momentum conservation law caused skeptical relation to Einsteinian theory of gravity of many well-known theorists from F. Klein to A.A. Logunov. Some of them proposed to replace GR by their own gravity theory in a flat space (for instance, [21]).

This situation remains actual and today. In accordance with the second Nöther theorem the local symmetry of the theory leads to appearance of identities between the extremals. 1)These identities determine the form of additional conditions decreasing the number of independent values in the equations and, so, permitting to solve them. 2)The Nöther identities open the way to geometrization of the given theory in terms of fibre bundle space geometry $[9,22]$.

Formally the second Nöther theorem and its generalization to Lie's group of the local internal symmetry, given by me in 1967 [10, 11], lead to a set of identities similar to the covariant derivative of left-hand sides of the motion equations. So, it is naturally that covariant conservation laws of sources will appear. And this is, really, so! The term in the Nöther identities, which is similar to the connection coefficient in the covariant derivatives of Riemannian space, contains the generator of Lie's algebra of symmetry group of the theory under discussion and corresponding structural constants. In electrodynamics the usual and above-mentioned covariant derivatives coincide because U(1)-Abelian group and its structural constants are equal to zero. Only in electrodynamics we can obtain the conservation law of the electric charge by means of both the first and the second Nöther theorems. But in spite of the similarity of these conservation laws, in the second case the current density will keep, but not the current.

By applying the second Nöther theorem to the theories with the non-Abelian symmetry group or non-vectorial variables we will have the identities, which need a special procedure for their integration. For non-gravitation fields it will integration on the fibre bundle space. In the case of GR we must integrate the covariant conservation law of the symmetric tensor of 
rank two, but not the vector, on the Riemannian space. Gauss theorem is not working in this case. In order to solve the problem of gravitation energy, the integration procedure proposed by Synge [23] can be applied. It is under discussion in the next subsection.

\subsection{Integral conservation laws for source currents. Geometry in large and in small}

What is the Earth surface similar to? The answer depends on the size of the region under consideration and precision of the instrument, which we have. The more is this region, the more corresponding surface is similar to spherical one. In a very small region around the fixed point the spherical surface is indistinguishable from the flat. Therefore both answers the Earth surface is spherical and the Earth surface is flat - may be right in dependence on the experiment conditions.

Each curved space can be regarded as a flat space in the small neighborhood of its point. So, every curved space can be regarded as a locally flat space. If the Riemannian space is covered by the flat spaces tangent in its every point, this construction will be called a tangent bundle. If instead of the tangent space in every point of the curved space a homogeneous space will be located, we shall obtain the fibre bundle space. The homogeneous space used instead of tangent space is named fibre. The initial curved space is named the base of the fibre bundle space.

Realization of this approach in GR consists in reformulation GR in terms of Cartan orthogonal frame of the reference connected with each point of Riemannian space-time being used by Einstein. In other words, we replace the curved Riemannian space by a set of flat Minkowski space-times associated with each point of the initial Riemannian space. In the case of electromagnetic, Yang-Mills and other gauge fields instead of Minkowski space-time we must use the homogeneous spaces with the corresponding local Lie gauge groups acting in these spaces.

In order to obtain integral conservation laws from differential one, we need to know the space configuration as a whole. Then we shall transform it into itself to study its global symmetry properties. But in the general case the Riemannian space as a whole does not have any symmetry properties. In accordance with Petrov classifications of Riemannian spaces [24] the symmetry properties of them (the motion groups) can be defined by the condition $\delta g_{\mu \nu}=0$. This condition permits to select that trajectories where the metric of Riemannian space remains without variation during the space transformations are fulfilling. These transformations are characterized by Killing vectors $\xi_{\mu}^{a}$, where $a$ is the motion group parameter.

In order to get the integral invariant values in GR, it is necessary to integrate the tensor energy-momentum components along the motion group trajectories of Riemannian spacetime. Really in Minkowski space-time we usually fulfill the similar procedure, but the Killing vectors are very simple (Kronecker delta) and we do not notice their presence. As a result in the Riemannian space-time we get a set of motion integrals whose number coincides with the number of the motion group parameters. These motion integrals form representation of algebra of the motion group of Riemannian space as a whole. It becomes clear that only in Minkowski space-time we shall have 10 motion integrals, forming the Poincare algebra of the motion group (i.e. when gravity absence). Another case when Riemannian space symmetry is defined by 10 motion integrals is the space of constant curvature. But then Killing vectors form the of Lie group algebra $\mathrm{O}(5)$ or $\mathrm{O}(4,1)$. So, it will be impossible to select energy as independent motion integral from the 5D-multiplet of the components forming the vector of energy-momentum-angular momentum. In all other cases the number of the motion integrals will be smaller and can become equal to zero [24]. In addition it is necessary to note that study of curved spaces as a whole implies knowledge of their topological properties, which are not connected with the space metric. 


\subsection{Einstein or Bohr. Who is right? Correspondence principle}

In 1918 Bohr formulated his correspondence principle [25]. It contained the statements, which influenced on attainment of agreement between physicists with respect to the problem: how to apply usual classical theory of radiation and quantum hypotheses to construction of atom models. In $1906 \mathrm{M}$. Plank was the first to show that in the limit $\hbar \rightarrow 0$ the results of quantum theory pass to the classical ones. In his opinion, the classical theory can be regarded as the theory, where action quantum is infinitely small. But Bohr regarded the correspondence principle as a key to explanation of the atom stability. In his approach the main things were two postulates: 1) orbit stationary state of electron motion, and 2) quantization of energy radiated by electron. So, it was postulated that electrons moving along the trajectories of planetary model of atom were not radiating energy. But at the moments of their jumps from one orbit to another the energy has radiating by portions (quantums). Bohr proposed that the energy quantity radiated in the jump processes is related with the frequency of electron inversion along the orbits by the following formula: $v_{\text {inv }}=v_{\text {rad }}$.

The Bohr atom model became generally recognized because it gave a correct explanation of nuclear spectra, and by means of it Bohr predicted a new element of D. Mendeleev's periodic elements system. This element was discovered in 1922 and named hafnium. The message about this discovery was received in Denmark when Bohr became Nobel Prizeman of 1922 for his investigations of the atom structure and radiation. Einstein became Nobel Prizeman in 1921.

The correspondence principle led Heisenberg to matrix formulation of quantum mechanics [26]. Let us apply this principle and Einsteinian postulates of GR to the atomic structure.

Instead of stationary Bohr's orbits it is necessary to consider the geodesic lines of the introatomic Riemannian space. The motion along the geodesic lines is inertial one and similar to the motion along the straight lines in the Newtonian mechanics. This motion is going on without any radiation, by definition. Therefore it is also stationary by definition. A new object in this picture - metric or metric field $g_{\mu \nu}$. The presence of this field inside the atom does not always lead to the space curvature. Criterion of the space curvature appearance is nonzero Riemannian curvature tensor $R_{\mu \nu \tau}^{\lambda}$. If this condition is fulfilled then the geodesic motion remains inertial. The passages from one geodesic trajectory to the other will be described by the equation of geodesic deviation. This equation shows that the distance between two infinitely close trajectories will always variable. The mathematical structure of the geodesic deviation equation is similar to the structure of the wave equation. It is possible that the secret of atomic spectra is related with the properties of the Riemannian curvature tensor, and "jumps" of electron happen in the points of the geodesic lines intersections.

Can one hear the shape of a drum? Many of modern mathematicians are seeking this problem solution. Apparently the young physicists will decide it. Now the new branch of mathematics arose: geometric quantization [27]. It is application of harmonic analysis in quantum statistical mechanics and quantum field theory.

\section{Conclusions. Atom constructed by Bohr}

From the historical point of view theoretical physics is a very young science. Really, the first professor of theoretical physics was M. Plank. Maxwell was professor of mathematical physics. In 1900 quantum physics was only born. Two events assisted in that: Plank's discovery of action $S$ quantization (1900) and Einsteinian hypothesis on light quantums (1905). But already in $1913 \mathrm{~N}$. Bohr proposed his model of atom, which is successfully working up to our days. This model very effectively combines classical and quantum physics. It unifies 


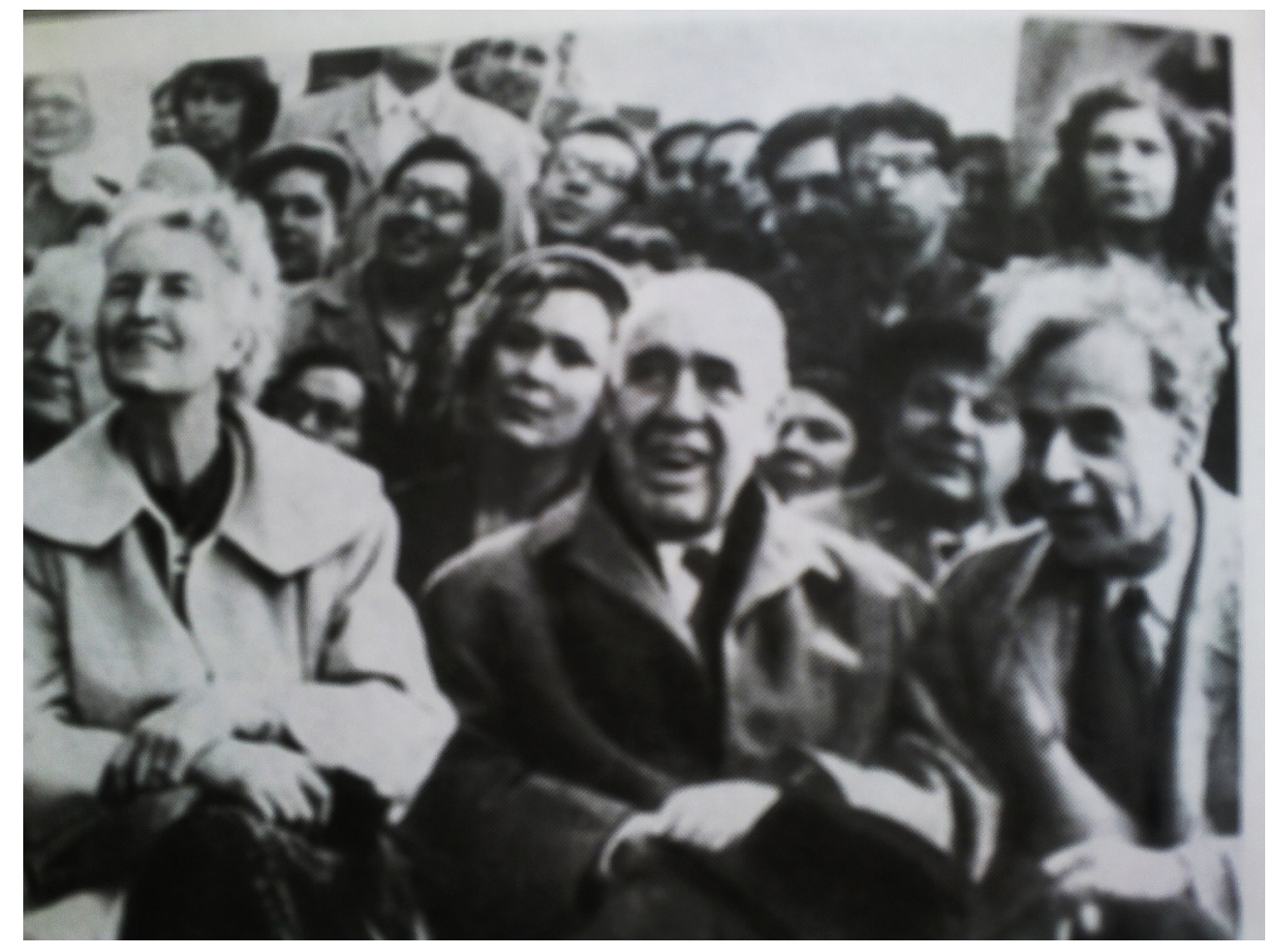

Figure 1. First row (sitting): Margrethe Bohr, Kora Landau, Niels Bohr, Lev Landau. Upper right corner (standing): Nelly Konopleva - student of the 3rd course of Physics Department of Moscow State University

micro- and macroworld representations. In 1965 M.A. Markov proposed some kind of elementary particle, inside which was universe [28]. This universe must be a closed universe of a very small radius for an exterior observer (of the order of Plank's length). Einstein did not like "quantum jumps" of Bohr. Above we proposed the method of elimination them from the Bohr atom by some statements of GR. But in 1913 GR did not exist yet!

When in 1961 Bohr visited Moscow State University the physicists celebrated Archimedes' Day [29]. Prof. D. Ivanenko, who in 1932 was the first to propose the protonneutron model of atomic nucleus, invited him to visit the theoretical physics Chair. On the Chair wall N. Bohr wrote: "Contraria non contrarictoria sed complementa sunt", i.e. "Contrasts are not contradictions, but complements". It is in accordance with the complementarity principle, which N. Borh formulated in 1927. This principle played an important role in formation of new methods of physical mentality. Once Bohr said: "If some statement is the absolute truth, then the opposite statement can be also the absolute truth". History of classical and quantum physics development in XX century gives us many examples of this kind situations. Atom constructed by Bohr is one of them.

In 1961 after performance on the steps of MSU physical faculty (see photo) Bohr listened the opera "Archimedes" created by the young physicists - students and graduate students of the physical faculty. For the first time this opera was played in 1960 at the Palace of Culture of MSU. After listening to this opera Bohr climbed to the stage and said: "... This 
opera contains so much fantasy and talent, that I am easy in my mind for future of the Soviet theoretical physics".

Acknowledgments. It is a pleasure to thank the Organizers of the International Seminar ISHEPP XXIV for their support of this investigation.

\section{References}

[1] M.A. Markov, in Proc. of the Intern. Seminar on Vector Mesons and Electromagnetic Interactions, JINR, Dubna (1969)

[2] R.P. Feynman, Acta Phys. Pol. 24, 697 (1963)

[3] B.S. DeWitt, Phys. Rev. 160, 1113 (1967)

[4] L.D. Faddeev and V.N. Popov, Phys. Lett. 25B, 29-30 (1967)

[5] S. Weinberg, Phys.Rev.Lett. 19, 1264 (1967)

[6] G. t'Hooft and M.T. Veltman, Nuclear Phys. 448, 189 (1973)

[7] M. Gell-Mann, Report CTSL-20, California Institute of Technology, March 15, 1961

[8] S.L. Glashow, M. Gell-Mann, Ann.of Phys. 15, 437 (1961)

[9] N.P. Konopleva, "Relativistic physics as geometry" (E2-2001-92, JINR, Dubna, 2001) $1-10$

[10] N.P. Konopleva, "Gravitation and the Theory of Relativity", Nos.4-5, (Kazan State University, Kazan, 1968) 67

[11] N.P. Konopleva, V.N. Popov, Gauge fields (Atomizdat, Moscow, 1972) [in Russian]

[12] N.P. Konopleva, V.N. Popov, Gauge fields (Energoatomizdat, Moscow, 1980) [in Russian]

[13] N.P. Konopleva, V.N. Popov, Gauge fields (Harwood Academic Publisher, ChurLondon-New York, 1981)

[14] N.P. Konopleva, V.N. Popov, Gauge fields (URSS, Moscow, 2000) [in Russian]

[15] A. Einstein, Sitz.Preuss.Acad.Wiss. 2, 1111-1116 (1916)

[16] H. Weyl, Gravitation und Elektrizität (Sitz. Preuss. Acad. Wiss., Berlin, 1918)

[17] H. Weyl, Z.Phys. 56, 330 (1929)

[18] W. Pauli, Rev. Mod.Phys. 13, 203-232 (1941)

[19] E. Nöther, Nachr.Gess.Wiss.Goettingen, Math.-Phys. Kl. 2, 235 (1918)

[20] F. Klein, Vergleichende Betrachtungen über neuere geometrische Vorschungen (A.Deichert, Erlangen, 1972)

[21] V.A. Fock, Theory of Space, Time and Gravitation (Pergamon Press, London, 1959)

[22] A. Lichnerowicz, Théorie Globale des Connexions et des Groupes d'Holonomie (Consiglio Nazionale delle Ricerche, Rome, 1955)

[23] J.L. Synge, Relativity: The General Theory (North-Holland Publishing Company, Amsterdam, 1960)

[24] A.Z. Petrov, New Methods in the General Theory of Relativity (Nauka, Moscow, 1966) [in Russian]

[25] N. Bohr, Collected Works v.3, The Correspondence Principle - (19181923)(Ed.J.R.Nielsen Amsterdam: North-Holland, 1976)

[26] M. Jammer, The conceptual development of Quantum Mechanics (Mc Graw Hill book Company, New York, St.Luuis, San Francisco, Toronto, London, Sydney, 1967)

[27] N.E. Hurt, Geometric Quantization in Action (D.Reidel Publishing Company, Dordrecht: Holland/Boston:U.S.A./London:England, 1983)

[28] M.A. Markov, Progr.Theor.Phys. Suppl. E 65, 85 (1965)

[29] Collection works, Niels Bohr and science of XX century (Naukova dumka, Kiev, 1988) 